\title{
Thyrotoxic storm during sepsis: study of a rare complication, clinical and biological observations
}

\author{
Ferdinando Dodi, ${ }^{1}$ Monica Centanaro, ${ }^{2}$ Elisa Porcile, ${ }^{2}$ Elisabetta Riccò, ${ }^{2}$ Anna Di Noto, ${ }^{2}$ \\ Angelo Gratarola ${ }^{2}$ \\ ${ }^{1}$ Department of Infectious Diseases, San Martino Hospital, Genoa; ${ }^{2}$ Department of Anesthesiology \\ and Reanimation, San Martino Hospital, Genoa, Italy
}

\begin{abstract}
We describe an uncommon severe clinical complication of sepsis thyrotoxic storm (TS). Common biologic, pathogenic, clinical conditions that can determine sepsis can cause a TS. High circulating values of vascular cellular adhesive molecules-1 and various pro-inflammatory citochines are related with thyroid damage. During sepsis, moderate dysfunctions are not expression of actual disease, but severe damage in pituitary-thyroid hormonal axis. Thyroid stimulated hormon release, and consequent overproduction of thyroid hormones can cause TS. In critical patients, presence of hormonal dysfunctions determines more elevated clinical score of sepsis severity and higher risk of death than control groups (without thyroid disease) or survived patients.
\end{abstract}

\section{Introduction}

Thyrotoxic storm (TS) is a clinical syndrome marked by strong adrenergic activation due to overproduction of triiodotironine (T3) and tiroxine (T4) and their metabolic effects. Pulmonary edema with depletion of circulating liquid volume, jaundice, renal insufficiency are manifestations of severe thyrotoxicosis. ${ }^{1}$ TS is related to every cause that can produce an excessive thyroid hormones concentration. ${ }^{2}$ If not

Correspondence: Ferdinando Dodi, Department of Infectious Diseases, San Martino Hospital, Largo R. Benzi 10, 16132 Genoa, Italy.

Tel: $+39.010 .5554662-+39.3299615085$.

E-mail: ferdinando.dodi@Hsanmartino.it

Conflict of interest: the authors declare no potential conflict of interest.

Key words: Thyrotoxic storm; Hyperthyroidism; Sepsis.

Received for publication: 28 June 2015.

Accepted for publication: 24 April 2016.

(c) Copyright F. Dodi et al., 2016

Licensee PAGEPress, Italy

Journal of Biological Research 2016; 89:5402

doi:10.4081/jbr.2016.5402

This article is distributed under the terms of the Creative Commons Attribution Noncommercial License (by-nc 4.0) which permits any noncommercial use, distribution, and reproduction in any medium, provided the original author(s) and source are credited. adequately treated, TS is fatal, but mortality rate varies from 20 to $50 \% .{ }^{1}$ In the general population, incidence of sub-acute or acute hyperthyroidism varies from 0.05 to $0.1 \%{ }^{1}$ TS appears in $10 \%$ of hospitalization for hyperthyroidism. ${ }^{2}$ Infectious diseases and resulting systemic damages due to sepsis can determine TS and are considered as common causes of TS. ${ }^{2,3}$ In compromised hosts hospitalized in intensive care unit (ICU) - where multiple causes that can simulate TS are present - its precocious identification is difficult. ${ }^{2}$ Literature data about its actual incidence in sepsis are poor. ${ }^{4}$ Iatrogenic cause, like drugs amiodarone, benzofuranic iodine-enriched (37\% of molecular pound), and anti-arrhythmic drug, can originate thyroid dysfunctions in 15-20\%. We describe a clinic case of TS during multidrug resistant (MDR) Klebsiella pneumoniae septic shock (SS) observed in ICU, in January 2012, and its biologic pathogenesis.

\section{Materials and Methods}

The patient, a 75-year-old woman with chronic atrial fibrillation treated with oral amiodarone (maintenance dose of $200 \mathrm{mg} / \mathrm{die}$, administered five days a week), had an elective video-laparoscopic cholecystectomy for cholelithiasis. Surgical complications immediately arose: choleperitoneum, biliary-intestinal fistula, secondary and tertiary stercorary peritonitis, subsequent abdominal major surgery. Simplified acute physiology score II and sequential organ failure assessment were respectively 61 (high risk of severe prognosis) and 8 (high risk of organs failure). She showed persistent clinical complications, recurrent severe nosocomial infections, caused by MDR Gram-negative strains and Cytomegalovirus pneumonia as immunocompromised hosts, and multiple organs disease. In March 2012 (35 ${ }^{\text {th }}$ day of hospital stay), despite previous normal thyroid function, during SS and ventilator acquired pneumonia caused by MDR $K$. pneumoniae, and vascular catheter-related bloodstream by methicillin-resistant Staphylococcus epidermidis, TS arose. Symptoms were violent fever $\left(41^{\circ} \mathrm{C}\right)$, neurologic and psychic excitement, hypertension $(180 / 100 \mathrm{mmHg})$, tachyarrhythmia $(150 / \mathrm{min}$ heart-beats), atrial fibrillation, severe respiratory insufficiency, congestive heart failure, glycemic decompensation (HbAlc 9.5\%), neutrophilic leukocytosis (with white blood cells $25,000 / \mathrm{mmc}$ and neutrophils $12,500 / \mathrm{mmc}$ ). Serum thyroid hormonal values were: thyroidstimulating hormone $(\mathrm{TSH})=0.00 \mathrm{mU} / \mathrm{L}$ (normal values $0.270-4.200$ $\mathrm{mU} / \mathrm{L}$ ), T3=20.9 ng/L (normal values $1.80-4.60 \mathrm{ng} / \mathrm{L}$ ), T4=35.4 ng/L (normal values 9.30-17 ng/L). Anti-thyroglobulin and anti-thyroperoxidasis antibodies were absent. Necrotizing hepatic damage progressively began [aspartate transaminase/alanine transaminase $=190 / 210 \mathrm{UI} / \mathrm{L}$ vs 41/45 UI/L baseline values (40/40 UI/L normal values), total/direct bilirubin=22, 3/13, $8 \mathrm{mg} \%$ vs $10,5 / 8,35 \mathrm{mg} \%$ 
baseline values (normal values 1.2/0.5 mg\%)]. Thyroid echography showed: parenchymal inhomogeneity and hypoechogenicity, anteriorposterior diameters of right and left lobe 15 and $12 \mathrm{~mm}$. Endovenous antibiotic therapy was administered: vancomycin $(1 \mathrm{~g} / 48 \mathrm{~h}$, with maintenance breaktrough plasmatic levels from 10 to $20 \mathrm{mg} / \mathrm{L}$ ), colistimethate colistin $(2,000,000 \mathrm{U} /$ bid $)$, meropenem ( $1 \mathrm{~g} /$ bid $)$ and tigecycline (100 mg/die loading dose and $50 \mathrm{mg} /$ bid maintenance dose), adjusting for kidney insufficiency. Therapy for TS, via nasogastric tube, was: prednisolone (30 $\mathrm{mg} /$ die $)$, propranolol $(40 \mathrm{mg} /$ die $)$ and thiamazole (60 $\mathrm{mg} /$ die $)$. General and clinical conditions remained poor and unchanged. In $65^{\text {th }}$ day of hospital stay, serologic hormonal examination evidenced: TSH $0.2 \mathrm{mU} / \mathrm{mL}$, T3 $4.9 \mathrm{ng} / \mathrm{mL}$, T4 $30.5 \mathrm{ng} / \mathrm{mL}$. Metabolic interactions between amiodarone and other administered drugs were not present. The patient survived the TS acute phase but died on her $154^{\text {th }}$ day in hospital because of all the clinical complications, including sepsis. Diagnosis of the described infections was posed according to the criteria of the American College of Chest Physician and Society of Critical Care Medicine. ${ }^{5}$

\section{Results and Discussion}

TS case as presented is very rare in anesthesiology and infectious diseases clinic experiences. Clinical deterioration and multi-organ failure were the background in which TS appeared. SS was considered the TS trigger, with subsequent onset of pituitary-thyroid axis severe damage, thyrotropine release, T3 and T4 overproduction, TSH suppression, altered negative feedback of thyroid functions. ${ }^{3,6}$ Intranuclear cellular biologic activity explains pathogenesis of TS adrenergic activation: density increase of beta-adrenergic target receptors, post-receptors signals modification, genic activation and transcription induction by molecules after hormonal deiodination, antibodies for TSH receptor. Pro-inflammatory cellular factors, released during SS, are responsible for the shared pathogenic support both for multi-organ failure, typical of SS and TS: interleukins (IL1b, IL-6), alpha-tumor necrosis factor (TNFa), vascular cellular adhesins-1 (VCAM-1). ${ }^{6}$ Many clinical and biological studies demonstrate these combined interactions. IL1b, IL6, and TNFa can directly alter thyroid functions with various grades of clinical expression and severity, and can activate humoral reactions as in other organs and systems. ${ }^{6,7} \mathrm{TNFa}$ is also the major pro-inflammation mediator active on hormonal and metabolic side. SS creates endothelial homeostasis alterations for vascular cellular adhesins-1, mostly VCAM-1, and can originate from phenotypic variation of endothelial cells towards a pro-inflammatory status. ${ }^{8}$ Active leukocytes or other specific immunity cells can produce VCAM-1 activation too. ${ }^{8}$ Therefore, as described in our case, systemic inflammatory response syndrome to SS, an excessive immunity response, anaphylaxis-like, which appears against various provoca- tive bacterial antigens, mostly Gram-negative strains lipopolysaccharides, provides an inflammatory reactions cascade.,7

\section{Conclusions}

We evaluated if amiodarone-induced thyrotoxicosis (AIT) could be another possible cause of TS in our described case. Between the two clinical forms of AIT, AIT type 2 is the most frequent and, generally, it causes thyroiditis in subjects previously undamaged. ${ }^{8}$ Amiodarone could have a role as cofactor in causing thyrotoxicosis during the onset of AIT type $2 .{ }^{9}$ However, we think that the principal causes of such a devastating TS were biologic and immunologic alterations due to SS, which, in described clinical history, had a greater pathogenic role. According to scientific literature present on the subject, TNFa and VCAM-1 could be the strongest activators of inflammation, shared mediators of thyroid and septic damage, therefore the most probable causes of the set of symptoms described.

\section{References}

1. Mc Keown NJ, Tews MC, Gossain VV, et al. Hyperthyroidism. Emerg Med Clinic N Am 2005;23:669-85.

2. Nayak B, Burman K. Thyrotoxicosis and thyroid storm. Endocrinol Metab Clin 2006;35:663-86.

3. Goldberg PA, Inzucchi SE. Critical issues in endocrinology. Clin Chest Med 2003;24:583-6.

4. Panzer C, Beazley R, Braverman L. Rapid preoperative preparation for severe hyperthyroid Graves' disease. J Clin Endocr Metab 2004 89:2142-44.

5. Bone RC, Balk RA, Cerra FB, et al. ACCP/SCCM Consensus Conference Committee. Definitions for sepsis and organ failure and guidelines for the use of innovative therapies in sepsis. Chest 2009;136:e28.

6. Angelousi AG, Karageorgopoulos DE, Kapaskelis AM, Falagas ME. Association between thyroid function tests at baseline and the outcome of patients with sepsis or septic shock: a systematic review. Eur J Endocrinol 2011;164:147-55.

7. Cannon JG. Cytokines and shock. In: Kimball ES, ed. Cytokines and inflammation. Boca Raton, FL: CRC Press; 1991. pp 307-9.

8. Laudes IJ, Guo RF, Riedemann NC, et al. Disturbed homeostasis of lung intercellular adhesion molecule-1 and vascular cell adhesion molecule-1 during sepsis. Am J Pathol 2004;164:1435-45.

9. Bogazzi F, Bartalena L, Martino R. Approach to the patient with amiodarone-induced thyrotoxicosis. J Clin Endocr Metab 2010, 95:2529-35. 